\title{
Optimized prediction of cognition based on brain morphometry across the adult life span
}

\author{
Angeliki Tsapanou, Yaakov Stern, Christian Habeck* \\ Cognitive Neuroscience Division, Department of Neurology, Columbia University, New York, NY, USA
}

\section{A R T I C L E I N F O}

\section{Article history:}

Received 4 November 2019

Received in revised form 25 March 2020

Accepted 16 April 2020

Available online 24 April 2020

\section{Keywords:}

Aging

Brain morphometry

Out-of-sample prediction

fluid reasoning

vocabulary

\begin{abstract}
A B S T R A C T
We mapped out the combined and unique contributions of 5 different biomarkers for 2 cognitive outcomes in cognitively healthy adults. Beside associations of biomarkers with cognition in the full experimental sample, we focused on how well any such associations would persist in held-out data. Three hundred thirty-five cognitively normal participants, 20-80 years older, were included in the study. $Z$-scores were computed for fluid reasoning and vocabulary. The following imaging data were included: regional brain volume, regional thickness, fractional anisotropy of white-matter tracts, volumes of select deep gray-matter regions, and global white-matter hyperintensity. Volume accounted for most of the variance in both cognitive domains. In out-of-sample data, fluid reasoning was best predicted by volumes, but vocabulary by the combination of all modalities. Although the predictive utility was better overall for older participants, the information gleaned relative to null models was less for older participants. An optimized set of brain biomarkers can thus predict cognition in out-of-sample data, to various degrees, for both fluid and crystallized intelligence.
\end{abstract}

(c) 2020 Elsevier Inc. All rights reserved.

\section{Introduction}

Although the cognitive consequences of aging are evident, less is known about the biological factors, and their synergies, that contribute to the cognitive changes. Specific in-vivo measured brain biomarkers (BMs), both biochemical and imaging, are indicators of specific brain changes that may accompany changes in cognitive status. Interestingly, high-dimensional pattern regression on medical images via machine learning has been used for higher estimation accuracy and better generalizability in quantifying and detecting spatially complex imaging patterns of pathology from medical scans (Wang et al., 2010). Studies have proposed multiple regression models to build linear models estimating changes in cognition from structural magnetic resonance imaging (MRI) scans (Duchesne et al., 2005, 2009). Thus, a thorough validation of the joint effect of BMs is required for understanding age-related cognitive differences.

There are some limitations in the existing studies examining BM's association with cognition. Most of the sample sizes were relatively small, or studies were focused on specific age groups (i.e., only older adults), or used only a brief cognitive examination tests

\footnotetext{
* Corresponding author at: Cognitive Neuroscience Division, Department of Neurology, Columbia University, 630 W 168th St, P\&S Box 16, New York, NY 10032, USA. Tel.: 212-305-0945; fax: 212-342-1350.

E-mail address: ch629@columbia.edu (C. Habeck).
}

(i.e., the Mini Mental State Examination), hampering the generalizability of the results. Existing studies also mainly examined individual or pre-chosen sets of BMs, for example, hippocampal volume only, based on prior correlations with both age and cognition. Thus, the association with cognition for a variety of BMs considered in conjunction has only been rarely examined. Some studies have explored how a specific set of BMs can explain variation in cognition in cognitively normal older adults. For example, Hedden et al. (2016) evaluated the predictive utility of a set of individual BMs, selected based on prior association with aging and cognition, with a similar analysis also having been reported by our team (Tsapanou et al., 2019). However, these BMs were not specifically derived for optimized predictive utility. A natural next step for us was to attempt to derive an optimized set of BMs and test its account of cognitive variance in held-out data. Such an approach need not sacrifice inferential rigor and can further test predictive utility, something that is not routinely attempted in typical BM research surveys.

In the current study, we initially examined the variance in cognition associated with specially derived predictive patterns from each brain modality separately. More precisely, the modalities we used were the following: brain volumes and thicknesses in 68 regions of interest (ROIs), fractional anisotropy (FA) and medial diffusivity (MD) in 18 white matter tracts, deep gray-matter volume in 14 ROIs (DEEP), and total white matter hyperintensity burden (WMH). We then also considered pooled estimates or "votes" of all single-modality predictions which could be more predictive than 
the best of their individual modality constituents, in order to examine whether each factor and modality provides unique information. Our quasi out-of-sample prediction of cognition (explained in detail in the Methods section) marks the main conceptual and algorithmic centerpiece of the current study. Although probing the association of BMs with cognition in the full experimental sample is a natural and important first step, we wanted to also examine how well any such associations would persist in held-out data. Standard statistical inference in a training sample might not fully inform about the predictive utility of a fitted model in held-out data. We checked such replication empirically in split-sample simulations.

For our simulations we were interested in several questions, which, to our knowledge, have not been systematically investigated before: (1) what are the differences in predictive utility for different BM modalities? (2) How well can cognitive abilities pertaining to fluid versus crystallized intelligence be predicted from structural brain markers, and are there differences? (3) Is age associated with the predictability of cognition from brain, and are there differences between fluid and crystallized intelligence?

Our a priori hypotheses for the predictive utility of BMs were broad: we expected gray-matter (GM) structural measures to show better utility than WMHs. For the dependence of predictive utility on the type of cognition, we expected that fluid intelligence would show a stronger dependence on brain structure, reasoning that crystallized intelligence by its nature as a proxy for cognitive reserve would be less dependent on "brain real estate." Similarly, we expected that age would be negatively associated with the success of predicting cognition from brain: higher age might be associated with greater prominence of individual differences and residual factors not captured reliably by brain markers.

\section{Methods}

Participants were recruited for 2 studies: The Reference Ability Neural Network (RANN) and the Cognitive Reserve (CR) study. The RANN study was designed to identify networks of brain activity uniquely associated with performance across adulthood for each of the 4 following reference abilities: memory, reasoning, speed of processing, and vocabulary (Habeck et al., 2016). The CR study was designed to elucidate the neural underpinnings of cognitive reserve and the concept of brain reserve (Stern, 2012). All participants were native English speakers, right-handed, with at least a fourth-grade reading level. In order to be included in the study, participants had to be also free of any major neurological or psychiatric conditions that could affect their cognition. Careful screening excluded participants with mild cognitive impairment (MCI) or dementia. A score equal or greater than 130 was required on the Mattis Dementia Rating Scale (Mattis, 1988) for the inclusion in the studies. Moreover, participants had to have no or minimal complaints on a questionnaire about their functionality (Blessed et al., 1968). Both RANN and CR studies have been approved by the Institutional Review Board of Columbia University. More detailed information about the 2 studies can be found in previous publications (Habeck et al., 2016, 2017; Razlighi et al., 2017; Stern, 2009, 2012; Stern et al., 2014). We selected participants who had complete data on the measures used in the current analyses.

\subsection{Imaging data}

We used data from structural and resting state functional T1 MRI, diffusion tensor imaging (DTI), and fluid-attenuated inversion recovery (FLAIR). All scans were acquired on the same 3.0 T Philips Achieva MRI scanner. We used regional brain volumes and thicknesses in 68, and FA and MD in major tracts, derived from the FreeSurfer (v5.1.0) software for human brain imaging analysis (http://surfer.nmr.mgh.harvard.edu/). We also used 14 deep GM regions and $\log$ transformed global WMH burden $(\log (\mathrm{WMH}+1))$ derived from the FMRIB software library.

\subsubsection{Volume and cortical thickness}

A T1-weighed magnetization-prepared rapid gradient-echo (MPRAGE) scan was used. The scans were acquired with echo time/repetition time (TE/TR) of $3 / 6.5 \mathrm{~ms}$, flip angle of $8^{\circ}$, in-plane resolution of $256 \times 256$, field of view (FOV) of $25.6 \times 25.6 \mathrm{~cm}$, and $165-180$ slices in axial direction with slice thickness/gap of $1 / 0 \mathrm{~mm}$. The FreeSufer software was used for the reconstruction of the T1 scans (Fischl et al., 2002, 2004). We used the volumes (in $\mathrm{mm}^{3}$ ) of 68 ROIs (thicknesses in $\mathrm{mm}$ ). Thickness in 68 cortical regions (in $\mathrm{mm}$ ) was computed using the standard FreeSurfer parcellation (Desikan et al., 2006).

We also used the volume of 14 deep GM volume regions: right-left cerebellar cortex, right-left thalamic cortex, right-left caudate, right-left putamen, right-left pallidum, right-left hippocampus, and right-left amygdala.

\subsubsection{FA and $M D$}

Two sets of DTI images were acquired with each set having 56 directions using these parameters: $b=800 \mathrm{~s} / \mathrm{mm}^{2}, \mathrm{TE}=69 \mathrm{~ms}$, $\mathrm{TR}=7645-7671 \mathrm{~ms}$, FOV $2.4 \times 22.4 \mathrm{~cm}$, flip angle $=90^{\circ}$, in-plane resolution $112 \times 112$ voxels, acquisition time 9 minutes 27 seconds, slice thickness $=2 \mathrm{~mm}$ (no gap), and 75 slices. The 2 data sets were then concatenated and processed with Tracts Constrained by Underlying Anatomy DTI, distributed as part of the FreeSurfer library, which produces 18 major white matter tracts, as described in previous publications (Li et al., 2018; Yendiki et al., 2011). All tracts were used as individual input variables.

\subsubsection{WMH burden}

FLAIR images for visualization of WMH were used with the following parameters: Repetition Time/ Inversion Time (TR/TI) (ms) 11,000/2800 TE (ms): 125, in-plane resolution $256 \times 189$, FOV $23.0 \times 17.96 \mathrm{~cm}$, and 30 slices with slice thickness/gap of $4 / 0.5 \mathrm{~mm}$. For the extraction of the WMH we used the Lesion Segmentation Tool, which is a toolbox for Statistical Parametric Mapping, able to segment T2 hyperintense lesions in FLAIR images. Lesions were segmented by the lesion growth algorithm (Schmidt et al., 2012) as implemented in the Lesion Segmentation Tool version 2.0.15 (www. statistical-modelling.de/lst.html) for Statistical Parametric Mapping. Following standard convention, WMH voxel counts were logtransformed (according to $\log (\mathrm{WMH}+1)$ ) (resulting in a normal distribution) and treated as a continuous variable, with higher values indicating greater $\mathrm{WMH}$ burden.

\subsubsection{Deep GM ROIs}

The analysis resulted in 14 ROIs, and was performed by using the FMRIB software library SIENAX.

\subsection{Neuropsychological evaluation}

Each participant underwent an extensive neuropsychological evaluation. From the neuropsychological battery, we derived 2 cognitive domains to use in our analysis-fluid reasoning and vocabulary - so that we cover the examination of both fluid and crystallized intelligence. The following tests were used: for fluid reasoning: Wechsler Adult Intelligence Scale matrix reasoning, letter-number sequencing, and block design test, total correct (Wechsler, 1997). For vocabulary: Wechsler Adult Intelligence Scale vocabulary test, the Wechsler Test of Adult Reading (Wechsler, 2001), and the American National Adult Reading Test, total correct (Grober and Sliwinski, 1991). Z-scores were computed for each 
cognitive task, and scores were transformed, and sign-reversed if necessary, such that a higher score indicates better performance.

\section{Statistical analysis}

Statistical analyses were performed using in-house code written in MATLAB R2017a. Analyses were performed across the whole group, aged $20-80$.

We derived predictive patterns for each of the cognitive domains and general cognition using each of the brain measures: (1) volume, (2) thickness, (3) FA, (4) deep GM volume, and (5) WMH burden, using principal component analysis (PCA) regression as described below. (6) We further produced a composite prediction scheme (= "vote"): combining the predictions of all brain measures by averaging. All single-modality predictions and the prediction scheme were applied to held-out data.

\subsection{Quasi out-of-sample prediction of cognitive function}

The main computational frame for generating predictions and assessing predictive utility in held-out data consisted of many iterations of split-sample assignments, also termed Monte-Carlo 5-fold cross-validation. The data were split randomly 1000 times into training sets of 240 participants, and test sets of 60 participants. Using PCA (explained below), a prediction of a cognitive outcome measure in the test set on the basis of model estimated in the training set was calculated, and the goodness of the prediction was recorded to track performance. We had 2 cognitive outcomes (fluid reasoning and vocabulary) and 5 data modalities (outlined above). In total, we thus ran 2 split-sample simulations in which all 5 input modalities were tested, with the computation of a "vote predictor" which simply averaged the predictions of all 5 input modalities. For tracking prediction performance, we computed the Predicted Residual Sum of Squares (PRESS) in the held-out data. In total, for each of the 2 simulations, we thus have 1000 PRESS values for 6 predictions, that is, 5 single-modality predictions, and 1 vote of all 5 brain modality predictions. The PRESS statistic was presented in box-plots showing medians and interquartile ranges.

For full disclosure, we speak of "quasi" rather than genuine replication; because data for both training and test sets were sampled from a limited pool, they were not independent across iterations.

\section{2. $P C A$ regression/SSM}

PCA regression, in a brain-imaging context termed SSM (Habeck, 2010; Habeck et al., 2010; Moeller et al., 1987; Strother et al., 1995), was used to develop predictive models for the cognitive outcomes. This approach has been used extensively by our laboratory in the past and is an obvious choice for a continuous prediction problem $R^{N} \rightarrow R$, where $N$ features are reduced to one prediction of a cognitive outcome.

Although a strict implementation of PCA/SSM assumes multiplicative variability, and correspondingly imposes a logarithmic transform on all input data prior to the PCA, we did not perform this for the current paper. A log-transform did not enhance prediction performance and led to a slightly worse result for white matter tract integrity; for that reason, we omitted it.

Assuming the training and test data as $\mathbf{Y}$ and $\mathbf{Z}$ (with the same number of features as rows, but different numbers of observations as columns) and the set of principal components (PCs), derived via PCA only from $\mathbf{Y}$, as $\mathbf{V}$ (with as many rows as $\mathbf{Y}$ and $\mathbf{Z}$, and as many columns as components), the estimation step of the linear prediction model in the training set can be written as a linear regression according to:

Estimation step in training sample : $\mathbf{C o g}=\left[\begin{array}{lll}\mathbf{Y}^{\prime} & \mathbf{V} & \mathbf{1}\end{array}\right] \boldsymbol{\beta}+\boldsymbol{\varepsilon}$.

$\boldsymbol{\beta}=\operatorname{pinv}\left(\left[\begin{array}{ll}\mathbf{Y}^{\prime} \mathbf{V} & \mathbf{1}\end{array}\right]\right) \operatorname{Cog}$

where 1 denotes an intercept term, and ' denotes matrix transposition, and $\operatorname{Cog}$ denotes the cognitive outcome in question (either fluid reasoning or vocabulary). This linear regression produces the regression weights $\boldsymbol{\beta}$ and pinv stands for the Moore-Penrose pseudoinverse transformation.

Next, the estimated model is used in the held-out data to predict the cognitive outcome:

Prediction step in replication sample : $\operatorname{Cog}^{*}=\left[\mathbf{Z}^{\prime} \mathbf{V} \mathbf{1}\right] \boldsymbol{\beta}$.

In short, we projected a set of PCs obtained from the training set into the training set to obtain subject scores of all PCs in the training set, and then performed a linear regression with the cognitive outcome of interest in the training set as the dependent variable. Next, the subject scores of the same PC set were obtained in the test data set, and the prediction model (=regression weights) was applied to generate a prediction. This prediction can then be compared to the actual values of the cognitive outcome of interest in the test data set. As mentioned before, prediction performance was assessed with the PRESS statistic. The lower PRESS, the better the prediction. PRESS is the mean squared error of the prediction in the held-out data:

PRESS $=\left\langle\left(\operatorname{Cog}-\operatorname{Cog}^{*}\right)^{2}\right\rangle$

where $\operatorname{Cog}$ and $\operatorname{Cog}{ }^{*}$ denote the actual and the predicted values of the cognitive outcome in the replication sample, and $<>$ denoted the sample average in the held-out data. There are as many PRESS values as iteration in the simulation, that is, 1000 .

The set of selected PCs in $\mathbf{V}$ was chosen via minimization of the Akaike information criterion (AIC). All contiguous sets 1, 1:2, 1:3, $1: 4, \ldots, 1: N$, were tried out in the training sample in $N$ regressions, where $N$ was the number of Eigen values $>1$. The best-fitting set $1: K$ was chosen for which AIC was minimal.

In contrast to group-level statistics like correlation, PRESS can be assessed on a single-participant level. However, PRESS is not independent of the variance in the outcome measure and in the BM. Lower variance might result in lower PRESS values by default, that is, even in null data. To correct for such an effect and estimate the true predictive utility of the $\mathrm{BM}$, for each iteration we also ran a "null" model, that is, a model where the dependent cognitive variable in the training set was randomized. Such a null model's PRESS in the held-out data can serve as a reference benchmark how much meaningful information can be gleaned from the modality in question about the cognitive outcome of interest. We computed the normalized PRESS as the ratio

Normalized PRESS $=$ PRESS (actual) $/$ PRESS (null) $<1$ (!)

which should be smaller than unity, and serves as an absolute measure how much information can be provided by the BM about the outcome: a ratio close to unity indicates that not much information can be provided by the BM compared to the null prediction. Normalized PRESS can now serve as statistic to compare the predictive utility different modalities or cognitive outcomes.

Fig. 1 explains PCA/SSM and our split-sample framework in more detail. 
A

\title{
PCA-Regression, Scaled Subprofile Modeling (SSM)
}

\author{
Step 1: PCA of biomarker data $\quad \mathbf{Y}=\mathbf{V} \mathbf{W}^{\prime}$ \\ $\mathbf{Y}=$ biomarker data,
$\mathbf{V}=$ principal components,
$\mathbf{W}=$ pattern scores \\ $[Y]=\#$ variables $\quad x \quad \#$ participants \\ $[\mathrm{V}]=$ \# variables $\mathrm{x} \quad$ \# components \\ $[$ W] $=\#$ participants $x \quad \#$ components
}

Step 2: Brain-behavioral regression

$$
\text { Cognition }=[W(:, 1: K) 1] * \beta+\varepsilon
$$

$\rightarrow$ Regression weights $\beta$ and best-fitting set of components 1:K are obtained with AIC criterion

Step 3: Application of brain behavioral model to new data $\mathbf{Z}$

Predicted cognition $=\left[Z^{\prime *} \mathrm{~V}(:, 1: K) 1\right] * \beta$

$\rightarrow$ Regression weights $\beta$ and PCs are used to predict cognitive outcome in new data

B

Split-sample simulations with SSM:

\section{Repeat $1,000 x$}

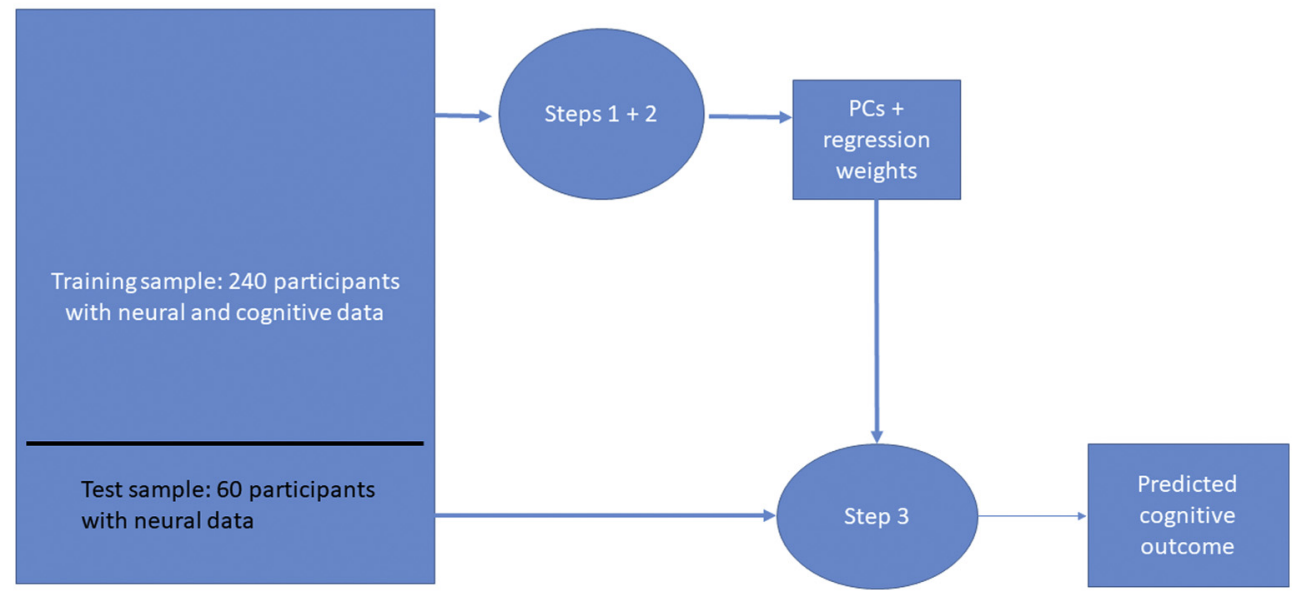

Fig. 1. Computational recipe for scaled subprofile modeling (A), and the split-sample framework used in the current article (B). Abbreviations: PCA, principal component analysis; SSM, scaled subprofile modeling.

\subsection{Composition of cognition-related patterns}

Prediction in independent data is a stringent and ecologically valid test for the diagnostic power of cognitive markers, but the composition of the associated BM pattern is of significant interest too. We checked whether the patterns were stable across different resampling iterations, that is, whether there were regions or tracts consistently featured in the derived patterns with a consistent sign. For visualization of pattern loadings, for every region or tract, we computed the empirical [1\%, 99\%] coverage interval. If the interval fell below/above zero, we assigned a negative/positive sign. The issue of pattern composition only applied to 4 modalities: (1) regional volume ROIs, (2) regional thickness ROIs, (3) tract-based measures, and (4) deep gray matter ROIs. For WMH and the composite "all brain" vote, no pattern is available.

\subsection{Relationship of prediction success to age}

A question of great interest to us was whether predictive utility shows differential associations to age. To avoid any loss of statistical power we refrained from (anyways somewhat arbitrary) age stratification and did not subset our data; however, we computed the mean age value for every replication sample. A simple bivariate correlation was run across all 1000 iterations to test whether the mean age in the replication sample was related to the normalized PRESS value.

\subsection{Distribution of PC sets in split-sample simulations}

We also explored the distribution of obtained PC sets for all 4 modalities on which PCA/SSM was performed. The maximum PC 
Table 1

Significant loadings at all 1000 patterns for each brain modality, age group, and cognitive outcome

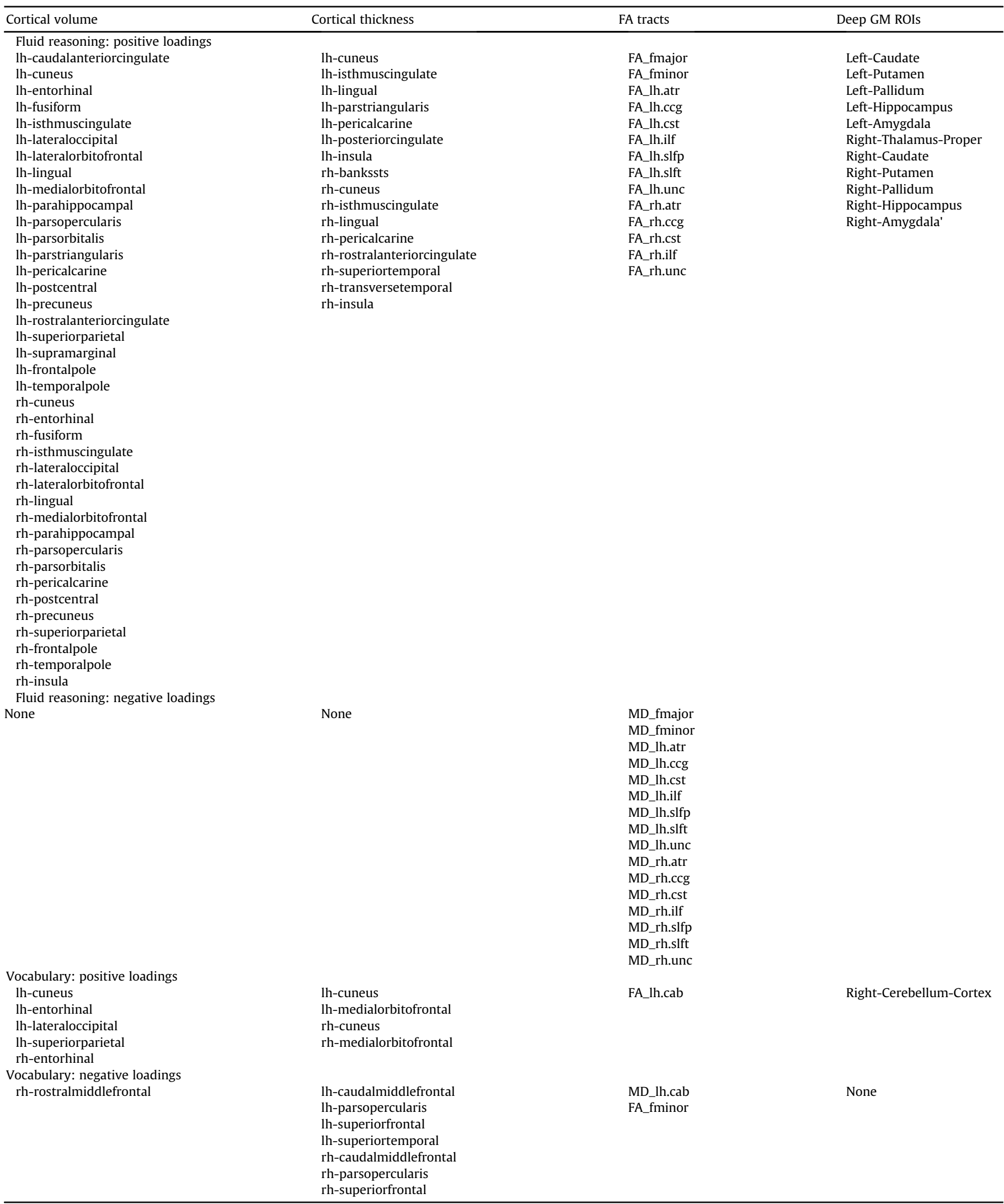

Presented are variables that have loadings with the coverage interval [1\%, 99\%] excluding zero.

Key: FA, fractional anisotropy; GM, gray matter; ROIs, regions of interest. 
Table 2

Summary of fit statistics for each modality and cognitive domain

\begin{tabular}{lll}
\hline & Fluid reasoning & Vocabulary \\
\hline Vol & 0.2223 & 0.1085 \\
Thx & 0.1148 & 0.1074 \\
Tracts & 0.0827 & 0.0517 \\
Deep GM & 0.1763 & 0.0285 \\
Log (WMH +1$)$ & 0.0312 & 0.0182 \\
All brain & 0.2495 & 0.2085 \\
\hline
\end{tabular}

The variance accounted for $\left(\mathrm{VAF}=R^{2}\right)$ in each cognitive outcome is tabulated. The models were run in the full group of participants: no replication in held-out data was performed. PCA/SSM was run for all modalities but WMH, for which simple linear regression was used.

Key: GM, gray matter; PCA, principal component analysis; SSM, scaled subprofile modeling; Thx, thickness; Vol, volume; WMH, white matter hyperintensity.

index, $K$, of the set $1: K$ was chosen according to the AIC. We plotted all distributions for all $2 \times 4$ predictions as histograms to convey a visual impression without further quantitative analysis.

\section{Results}

\subsection{Demographic characteristics/correlations}

The sample consisted of 335 participants. There were slightly more women (57.6\%), mean age was 51 years (standard deviation 16.5), and mean education was 16 (standard deviation 2.3) years, with a range of $12-22$ years. The PCA-based derivation of brainbased markers of cognition revealed significant associations between the 2 cognitive domains and each of the brain modalities. In Table 1, we present the significant loadings across all 1000 patterns for each brain modality and cognitive outcome. Positive loadings were observed for all 4 modalities for fluid reasoning, while only tract-based medial diffusivity measures showed negative loadings. For vocabulary, most modalities showed negative as well as positive loadings.

\subsection{Direct relationship of brain markers to cognitive domains}

The variance accounted for both fluid reasoning and vocabulary by the brain modalities is tabulated in Table 2 .

\subsection{Prediction success}

In Figs. 2 and 3, we present the prediction success for both cognitive outcomes as quantified by the median and interquartile ranges for the PRESS and normalized PRESS statistic. In general, the actual prediction was better than the null prediction. Only for $\mathrm{WMH}$, there is no real difference between actual and null prediction: for both cognitive domains, more than $26 \%$ of normalized PRESS values for WMH lie above unity for both outcomes.

Fluid reasoning was better predicted by volumes, followed by the combination of all brain modalities. Vocabulary was better predicted by the combination of all brain modalities, followed by the volumes. Overall, fluid reasoning was much better predicted than vocabulary.

\subsection{Relationship of age to prediction success}

We also computed bivariate correlations of the PRESS and normalized PRESS value with the mean age in the replication samples for both simulations (Tables 2 and 3). Although there were no findings for vocabulary, fluid reasoning presented significant correlations for most marker modalities: PRESS was negatively associated with the mean sample age for all 6 predictions. However, for a majority of the predictions, the normalized PRESS value was associated positively with mean sample age. The null models themselves become better as the replication sample age increases. Thus, while the apparent predictive utility improves overall for older participants, the information gleaned relative to null models becomes less for older participants.

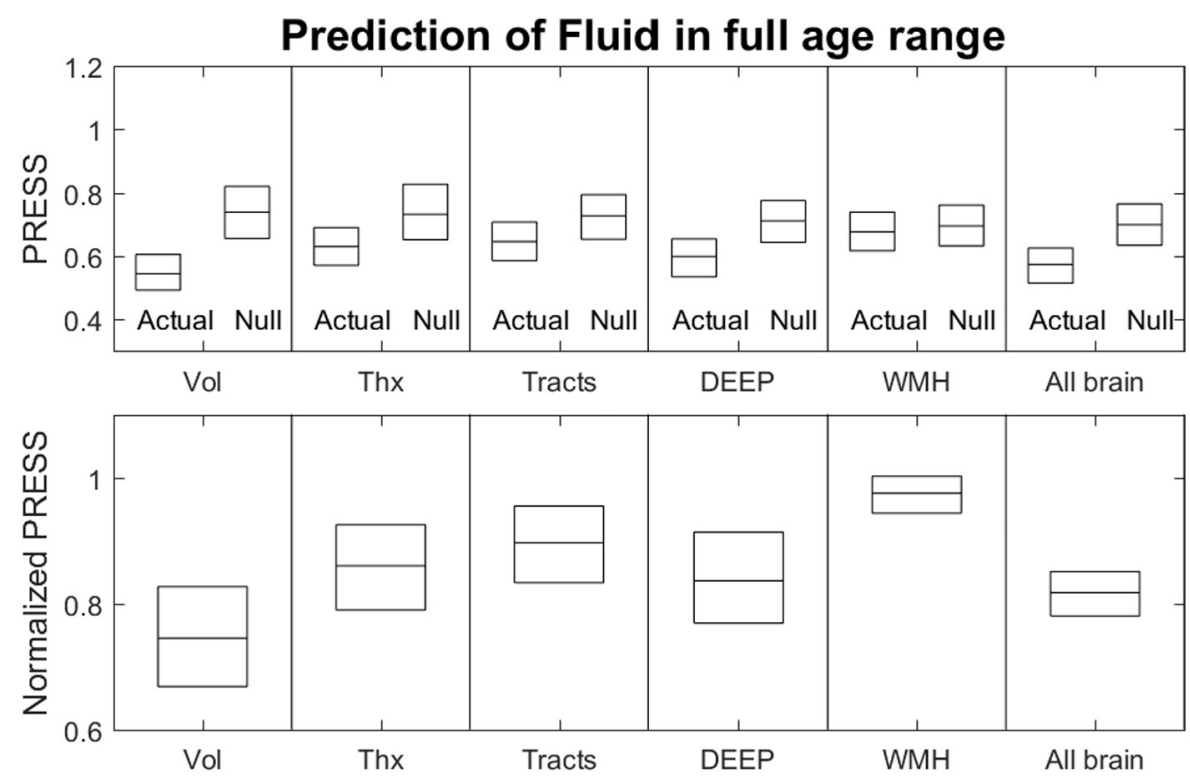

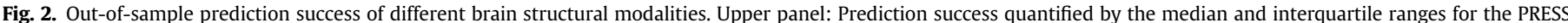

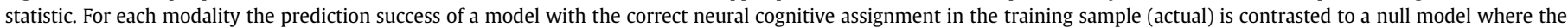

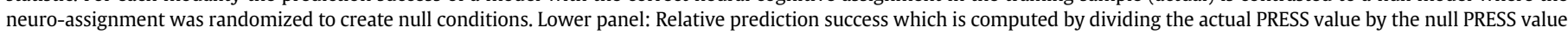

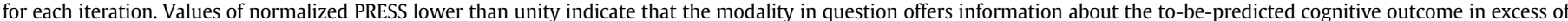

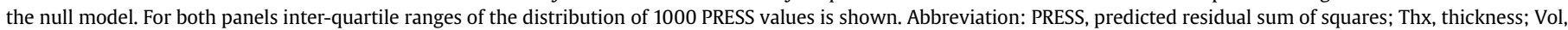
volume; WMH, white matter hyperintensity. 

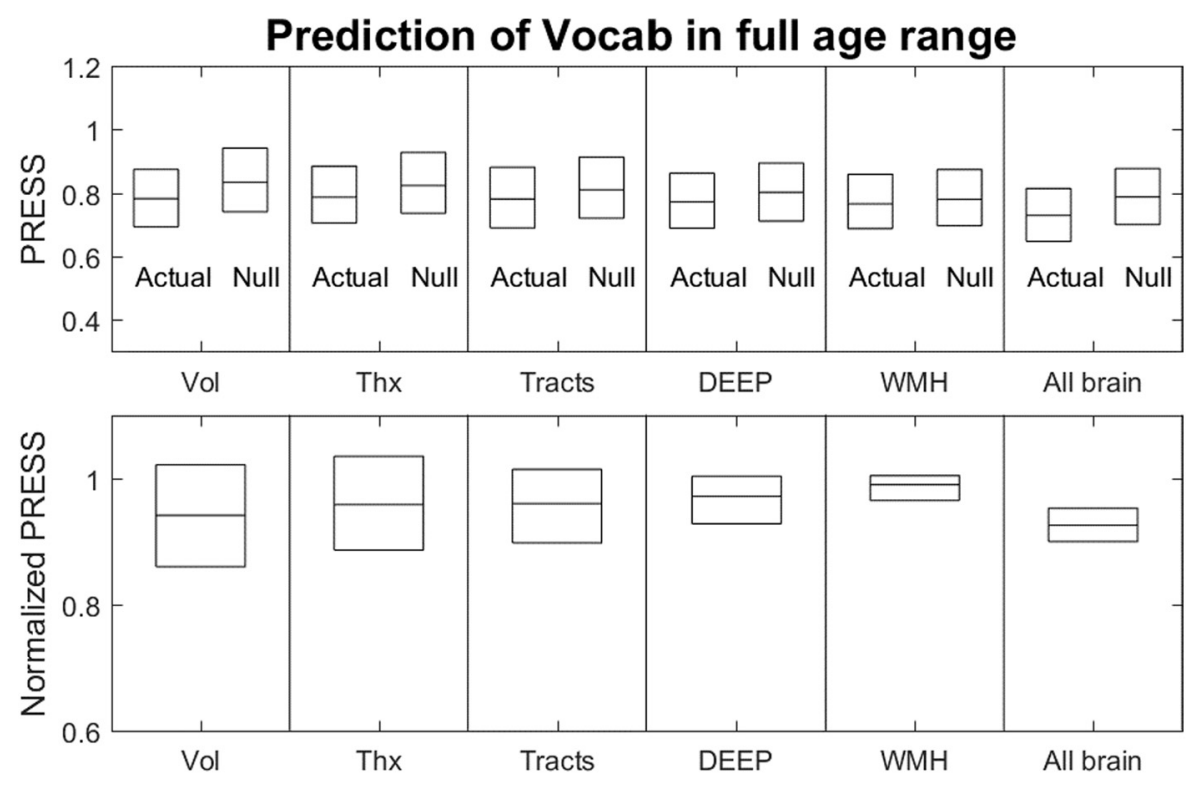

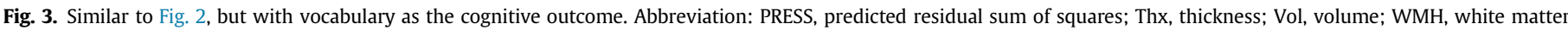
hyperintensity.

\subsection{Distribution of PC sets in split-sample simulations}

Fig. 4 shows the distribution of the maximum PC index, $K$, of the chosen PC sets $1: K$ in our split-sample simulations.

Consistent with the better prediction success for fluid reasoning, the figure shows better variance concentration with lower $\mathrm{K}$ values for fluid reasoning compared to vocabulary for all modalities but the deep GM ROIs. Performing PCA/SSM in the training sample often involves a larger set of PCs for vocabulary compared to fluid reasoning, resulting in fewer robust loadings, consistent with worse out-of-sample replication.

\section{Discussion}

We examined the association of an optimized set of brain structural modalities with cognition in cognitively healthy adults across a wide age range. We took a step further and examined how

Table 3

Bivariate Pearson correlation coefficients among PRESS, normalized PRESS, and mean age in the replication sample

\begin{tabular}{lll}
\hline & PRESS & Normalized PRESS \\
\hline Fluid reasoning prediction & & \\
Volumes & $-0.1287^{\mathrm{a}}$ & $0.1092^{\mathrm{b}}$ \\
Thickness & $-0.1308^{\mathrm{a}}$ & $0.1450^{\mathrm{a}}$ \\
Tract-based measures & $-0.1941^{\mathrm{a}}$ & 0.0295 \\
Deep gray matter ROIs & $-0.1310^{\mathrm{a}}$ & $0.1274^{\mathrm{b}}$ \\
WMH & $-0.2090^{\mathrm{a}}$ & $0.0702^{\mathrm{b}}$ \\
All brain & $-0.1936^{\mathrm{a}}$ & $0.1301^{\mathrm{a}}$ \\
Vocabulary prediction & & \\
Volumes & 0.0207 & -0.0317 \\
Thickness & 0.0547 & -0.0059 \\
Tract-based measures & 0.0168 & -0.0396 \\
Deep gray matter ROIs & 0.0413 & 0.0428 \\
WMH & 0.0172 & $-0.0661^{\mathrm{c}}$ \\
All brain & 0.0296 & -0.0277 \\
\hline
\end{tabular}

Key: PRESS, predicted residual sum of squares; ROI, region of interest; $\mathrm{WMH}$, white matter hyperintensity.

${ }^{\mathrm{a}} p<0.0001$.

b $p<0.001$.

c $p<0.05$. well these associations predicted performance in out-of-sample data. Our paper put the practical aim of prediction in held-out data, that is, inference of unknown from known information with models estimated in prior data, directly to the test. Surface and deep GM volumes accounted for most of the variance in both cognitive domains. In the out-of-sample analyses, both fluid reasoning and vocabulary performance were best predicted by volumes and the combination of all brain modalities, respectively.

For both cognitive domains, it is noteworthy that out of all the individual brain modalities, volume had the highest predictive utility. Existing longitudinal studies highlight the importance of the brain volume to predict cognition, $\mathrm{MCI}$, or dementia in older adults (Csernansky et al., 2005). Regional brain atrophy in MCI patients has been also reported to predict subsequent cognitive decline (Fan et al., 2008). Thus, our results are in accordance with prior investigations regarding the strong association between brain volume and cognition.

Somewhat surprising was the finding that predictive success (implying low PRESS) in the held-out data was positively associated with the mean age in the replication sample. We had anticipated the exact opposite: for example, younger adults have more robust networks and more efficient connectivity than the older ones (Bishop et al., 2010; Stern et al., 2005). Moreover, we hypothesized that older adults may be using unique strategies honed over a lifetime, contrary to the more anatomically specific younger brains. This finding stresses the importance of normalizing PRESS using null prediction data. The information provided above and beyond the null prediction, as quantified by normalized PRESS, was lower for higher mean sample age. Pragmatically, predicting cognition for older participants might work better than for younger participants, although not because of better mechanistic information provided by the BMs, but by virtue of generically reduced variance that would be even be true for null data. The same consideration might recommend always forming multi-modal averages of predictions as in the "all brain" vote: from Figs. 2 and 3, the reader can appreciate that both PRESS values (i.e., for the correct and randomized neurocognitive assignment in the training sample) are the lowest for the vote predictor, hinting that averaging null predictions might appear to be advantageous if it results in reduced variance. 

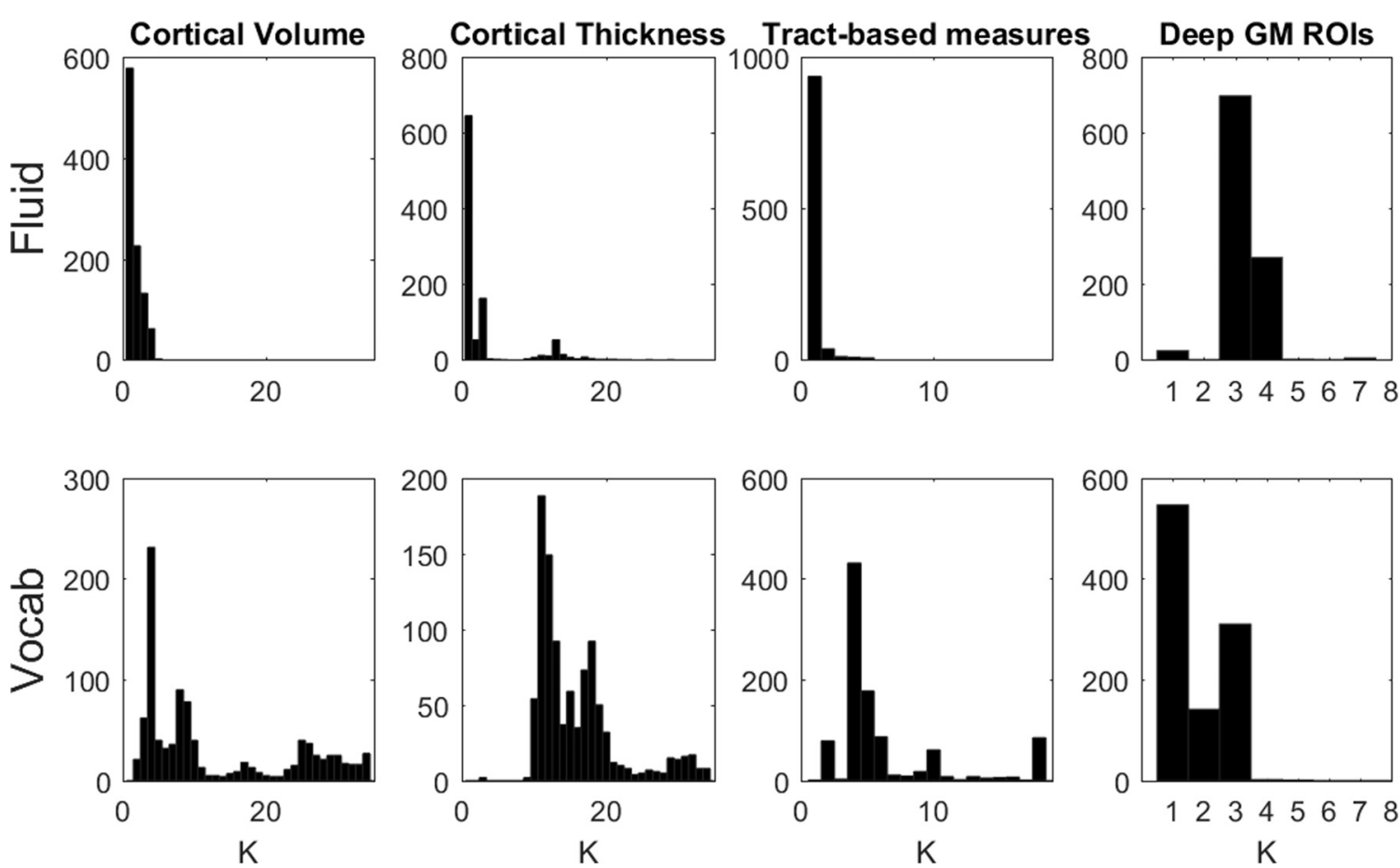

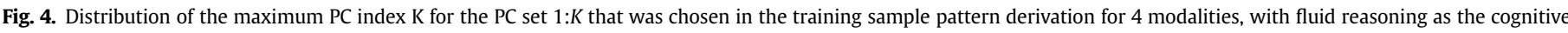

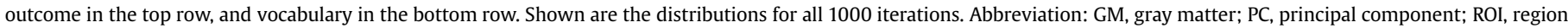
of interest.

Concerning prediction success by cognitive outcome, we observed that crystallized cognition was less well determined by structural brain markers than fluid cognition. Cognitive reserve which has been significantly associated with aging (Stern, 2009, 2012; Tucker and Stern, 2011) could play a significant role to this association. We could hypothesize that cognitive reserve is more active in cognitively normal older adults than the younger ones, "suppressing" the predictive cognitive ability of brain factors. Thus, we would expect that factors such as education, IQ and occupational attainment would have greater cognitive predictive utility in the older population. Finally, although our sample consisted of cognitively normal participants without any major neurological or psychiatric diseases, it is possible that non-pathological or pathological brain changes related to normal aging could increase brain and cognitive variability in older brains, limiting the out-of-sample predictability. Our analytic framework assumes group invariant patterns. Increased inter-individual variability makes this assumption, and thus successful prediction, less tenable.

The cross-sectional design is a main limitation of the study. Furthermore, the choice of the specific predictors neglected other, potentially also important factors (i.e., personality or genetics) with likely contributions to cognitive variation. Our approach, while aimed at out-of-sample replication with a simple enough multivariate approach, is not very common in the literature. It is conceivable that further machine-learning sophistication could achieve better prediction in out-of-sample data, using combinations and whole ensembles of deeper learning architectures, rather than employing a flat "one shot" PCA regression. For the current paper, we wanted to lay the ground with this simple non-iterative analytic framework, using a technique of familiarity in our laboratory.

Finally, the sample size for each analytical group was relatively small, limiting the statistical power of the analyses.

To our knowledge, this is the first study to examine a variety of brain modalities and derive patterns to explain maximal variance in cognition. Here, rather than focusing on pre-defined brain features, we examined the association between BMs and cognition by tailoring best-predicting patterns from an expanded number of BMs in a flexible, yet inferentially rigorous, manner. We are not aware of another study that included tests of predictive utility in held-out data. We also used 2 cognitive domains instead of one general cognitive score, to better hone in on the biomarker patterns underying fluid and crystallized intelligence and their possible differences.

In summary: both fluid and crystallized intelligence can be predicted in held-out data by an optimized set of brain morphometric markers, in cognitively healthy adults. The achieved prediction success is compellingly larger than what would be achieved a random "null" prediction. The added diagnostic potential of such multi-modal markers for upcoming cognitive decline or neurodegeneration will have to be assessed rigorously in longitudinal data sets. Our study marks a first step in this regard.

\section{Disclosure statement}

The authors have nothing to disclose.

\section{CRediT authorship contribution statement}

Angeliki Tsapanou: Conceptualization, Methodology, Writing original draft. Yaakov Stern: Conceptualization, Methodology, Writing - original draft. Christian Habeck: Conceptualization, Methodology, Writing - original draft, Formal analysis, Supervision.

\section{Acknowledgements}

This work was supported by the National Institute of Health (NIH)/National Institute of Aging (NIA) (grant numbers R01 AG026158 and RF1 AG038465). 


\section{References}

Bishop, N.A., Lu, T., Yankner, B.A., 2010. Neural mechanisms of ageing and cognitive decline. Nature 464, 529-535.

Blessed, G., Tomlinson, B.E., Roth, M., 1968. The association between quantitative measures of dementia and of senile change in the cerebral grey matter of elderly subjects. Br. J. Psychiatr. 114, 797-811.

Csernansky, J.G., Wang, L, Swank, J., Miller, J.P, Gado, M., McKeel, D., Miller, M.L. Morris, J.C., 2005. Preclinical detection of Alzheimer's disease: hippocampal shape and volume predict dementia onset in the elderly. Neuroimage 25, 783-792.

Desikan, R.S., Segonne, F., Fischl, B., Quinn, B.T., Dickerson, B.C., Blacker, D., Buckner, R.L., Dale, A.M., Maguire, R.P., Hyman, B.T., Albert, M.S., Killiany, R.J., 2006. An automated labeling system for subdividing the human cerebral cortex on MRI scans into gyral based regions of interest. Neuroimage 31, 968-980.

Duchesne, S., Caroli, A., Geroldi, C., Collins, D.L., Frisoni, G.B., 2009. Relating one-year cognitive change in mild cognitive impairment to baseline MRI features. Neuroimage $47,1363-1370$.

Duchesne, S., Caroli, A., Geroldi, C., Frisoni, G.B., Collins, D.L., 2005. Predicting clinical variable from MRI features: application to MMSE in MCI. Med. Image Comput. Comput. Assist. Interv. 8, 392-399.

Fan, Y., Batmanghelich, N., Clark, C.M., Davatzikos, C., Initiative Alzheimer's Disease Neuroimaging, 2008. Spatial patterns of brain atrophy in $\mathrm{MCI}$ patients, identified via high-dimensional pattern classification, predict subsequent cognitive decline. Neuroimage 39, 1731-1743.

Fischl, B., Salat, D.H., Busa, E., Albert, M., Dieterich, M., Haselgrove, C., van der Kouwe, A., Killiany, R., Kennedy, D., Klaveness, S., Montillo, A., Makris, N., Rosen, B., Dale, A.M., 2002. Whole brain segmentation: automated labeling of neuroanatomical structures in the human brain. Neuron 33, 341-355.

Fischl, B., Salat, D.H., van der Kouwe, A.J., Makris, N., Segonne, F., Quinn, B.T., Dale, A.M., 2004. Sequence-independent segmentation of magnetic resonance images. Neuroimage 23 (Suppl 1), S69-S84.

Grober, E., Sliwinski, M., 1991. Development and validation of a model for estimating premorbid verbal intelligence in the elderly. J. Clin. Exp. Neuropsychol. 13, 933-949.

Habeck, C.G. 2010. Basics of multivariate analysis in neuroimaging data. J. Vis. Exp.

Habeck, C., Gazes, Y., Razlighi, Q., Steffener, J., Brickman, A., Barulli, D., Salthouse, T., Stern, Y., 2016. The Reference Ability Neural Network Study: life-time stability of reference-ability neural networks derived from task maps of young adults. Neuroimage $125,693-704$

Habeck, C., Razlighi, Q., Gazes, Y., Barulli, D., Steffener, J., Stern, Y., 2017. Cognitive reserve and brain maintenance: orthogonal concepts in theory and practice. Cereb. Cortex 27, 3962-3969.

Habeck, C., Stern, Y., Initiative Alzheimer's Disease Neuroimaging, 2010. Multivariate data analysis for neuroimaging data: overview and application to Alzheimer's disease. Cell Biochem. Biophys. 58, 53-67.
Hedden, T., Schultz, A.P., Rieckmann, A., Mormino, E.C., Johnson, K.A., Sperling, R.A. Buckner, R.L., 2016. Multiple brain markers are linked to age-related variation in cognition. Cereb. Cortex 26, 1388-1400.

Li, P., Tsapanou, A., Qolamreza, R.R., Gazes, Y., 2018. White matter integrity mediates decline in age-related inhibitory control. Behav. Brain Res. 339, 249-254.

Mattis, S., 1988. Dementia Rating Scale (DRS). Psychological Assessment Resources, Odessa, FL.

Moeller, J.R., Strother, S.C., Sidtis, J.J., Rottenberg, D.A., 1987. Scaled subprofile model: a statistical approach to the analysis of functional patterns in positron emission tomographic data. J. Cereb. Blood Flow Metab. 7, 649-658.

Razlighi, Q.R., Habeck, C., Barulli, D., Stern, Y., 2017. Cognitive neuroscience neuroimaging repository for the adult lifespan. Neuroimage 144, 294-298.

Schmidt, P., Gaser, C., Arsic, M., Buck, D., Forschler, A., Berthele, A., Hoshi, M., Ilg, R. Schmid, V.J., Zimmer, C., Hemmer, B., Muhlau, M., 2012. An automated tool for detection of FLAIR-hyperintense white-matter lesions in Multiple Sclerosis. Neuroimage 59, 3774-3783.

Stern, Y., 2009. Cognitive reserve. Neuropsychologia 47, 2015-2028.

Stern, Y., 2012. Cognitive reserve in ageing and Alzheimer's disease. Lancet Neurol. 11, 1006-1012.

Stern, Y., Habeck, C., Moeller, J., Scarmeas, N., Anderson, K.E., Hilton, H.J., Flynn, J. Sackeim, H., van Heertum, R., 2005. Brain networks associated with cognitive reserve in healthy young and old adults. Cereb. Cortex 15, 394-402.

Stern, Y., Habeck, C., Steffener, J., Barulli, D., Gazes, Y., Razlighi, Q., Shaked, D. Salthouse, T., 2014. The Reference Ability Neural Network Study: motivation, design, and initial feasibility analyses. Neuroimage 103, 139-151.

Strother, S.C., Anderson, J.R., Schaper, K.A., Sidtis, J.J., Liow, J.S., Woods, R.P., Rottenberg, D.A., 1995. Principal component analysis and the scaled subprofile model compared to intersubject averaging and statistical parametric mapping: I. "Functional connectivity" of the human motor system studied with [150] water PET. J. Cereb. Blood Flow Metab. 15, 738-753.

Tsapanou, A. Habeck, C. Gazes, Y., Razlighi, O. Sakhardande, J., Stern, Y. Salthouse, T.A., 2019. Brain biomarkers and cognition across adulthood. Hum. Brain Mapp. 40, 3832-3842.

Tucker, A.M., Stern, Y., 2011. Cognitive reserve in aging. Curr. Alzheimer Res. 8 , $354-360$.

Wang, Y., Fan, Y., Bhatt, P., Davatzikos, C., 2010. High-dimensional pattern regression using machine learning: from medical images to continuous clinical variables. Neuroimage 50, 1519-1535.

Wechsler, D., 1997. Wechsler Adult Intelligence Scale, third ed. Harcourt Assessment, San Antonio, TX, pp. 684-690.

Wechsler, D., 2001. The Wechsler Test of Adult Reading (WTAR): Test Manual. Psychological Corporation, San Antonio, TX.

Yendiki, A., Panneck, P., Srinivasan, P., Stevens, A., Zollei, L., Augustinack, J., Wang, R. Salat, D., Ehrlich, S., Behrens, T., Jbabdi, S., Gollub, R., Fischl, B., 2011. Automated probabilistic reconstruction of white-matter pathways in health and disease using an atlas of the underlying anatomy. Front. Neuroinform. 5, 23. 